and PRO-C2), aggrecanase degraded aggrecan (AGNxI) and MMP degraded aggrecan (exFFGV). C1M and C3M are synovial membrane biomarkers and C2M, AGNxI and exFFGV are cartilage biomarkers.

Results: Explants were viable throughout the experiments, albeit the bSME lost some viability with time. bSME treated with $\mathrm{O}+\mathrm{T}$ showed increased $\mathrm{C} 1 \mathrm{M}$ and C3M ( $>400 \%$ and $>200 \%$ ) from day 10 , compared to $\mathrm{w} / \mathrm{o}$, whereas in bCC O+T increased C1M from day 21 and C3M from day $14(>800 \%,>1900 \%)$. O+T treatment increased C2M was increased from day 21 ( $>400 \%$ and $>1000 \%)$ in both BEX and bCC. The release was blocked by the generic MMP inhibitor GM6001 which also decreased the $\mathrm{C} 1 \mathrm{M}$ and C3M compared to w/o. O+T treatment increased AGNxl at day 7 and $10(>600 \%)$ and exFFGV from day 21 (>650\%) in both BEX and bCC. In bSME, TGF-b1 continuously and dose-dependently increased P1NP from day 7 compared to w/o (250\%). O+T pre-treatment for 10 days followed by TGF-b1 stimulation increased P1NP after 7 days of TNF-b1 treatment (150\%, figure). IGF-1 did not affect the P1NP level at any time point in bSME. In bCC both TGF-b1 (dose-dependently) and IGF-1 sustained the PRO-C2 level at the level of baseline throughout the study periods (figure), whereas $\mathrm{O}+\mathrm{T}$ decreased PRO-C2 compared to with w/o. The PRO-C2 level in BEX with TGF-b1 was unaltered compared to $\mathrm{w} / \mathrm{o}$.

Formation of type I collagen in bSME

after 10days catabolic stimulation with $\mathrm{O}+\mathrm{T}$

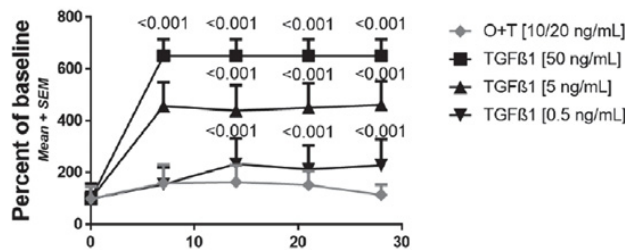

Formation of type II collagen in bCc

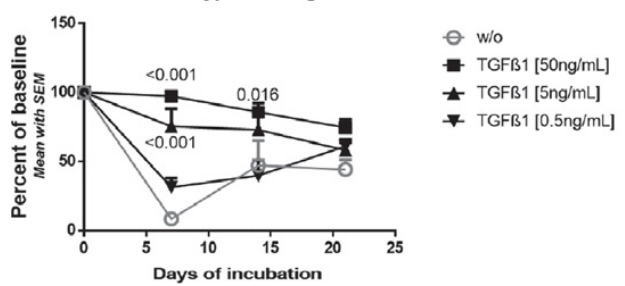

Conclusions: We here show that bovine synovium can be anaobolic and catabolic stimulated, both alone and in co-culture with cartilage. Anabolic stimulation was achieved with TGF-b1 in both bSME and bCC, while IGF-1 only showed an anabolic effect in the bCC. Previous human explant models using human tissuehave lacked the anabolic capacity. These translational explants models may be applied in the early development of anabolic drugs for cartilage degenerative diseases.

Disclosure of Interest: A. S. Siebuhr Employee of: Nordic Bioscience, C. Cabanes: None declared, S. Dahab: None declared, M. Karsdal Shareholder of: Nordic Bioscience, Employee of: Nordic Bioscience, A.-C. Bay-Jensen Employee of: Nordic Bioscience, C. Thudium Employee of: Nordic Bioscience DOI: 10.1136/annrheumdis-2017-eular.5737

\section{FRI0004 14-3-3ETA AS A NOVEL INVADOSOME REGULATORY MOLECULE IN RHEUMATOID ARTHRITIS}

C. Lalanne ${ }^{1}$, R.R. Lavoie ${ }^{1}$, M. Charbonneau ${ }^{1}$, J. Savill ${ }^{2}$, A. Marotta ${ }^{2}$, C. Dubois ${ }^{1}$. ${ }^{1}$ University of Sherbrooke, Sherbrooke; ${ }^{2}$ Augurex Life Sciences Corp, Vancouver, Canada

Background: Progressive cartilage destruction, mostly mediated by invasive fibroblast-like synoviocytes (FLS), is a central feature in the pathogenesis of rheumatoid arthritis (RA). We have reported that the ability of arthritic FLS to degrade the extracellular matrix depends on the formation of actin-rich plasma membrane invadosomal structures detected in cells strategically located at the cartilage-synovial membrane interface. Interference with the formation of invadosomes in RA FLS impeded matrix degradation in vitro and cartilage degradation in a model of collagen-induced arthritis, suggesting that invadosomes are important physiological structures involved in cartilage destruction.

The chaperonin molecule $14-3-3 \eta$ has been detected in the joints of patients with early and established RA and that its concentration in both serum and synovial fluid correlated with elevated expression of extracellular matrix (ECM)

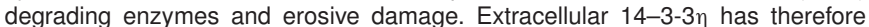
been proposed to be a novel biomarker for joint damage and a potential drug target for the personalized treatment of connective tissue-associated diseases but the direct relationship between $14-3-3 \eta$ and joint damage remains a key area of research.

Objectives: To evaluate the role of $14-3-3 \eta$ in the ability of synoviocytes to degrade the ECM.

Methods: mRNA from primary synoviocytes of healthy individuals $(\mathrm{N}=3)$ and $\mathrm{RA}$ patients $(\mathrm{N}=5)$ was extracted and the relative level of $14-3-3$ isoforms and MMP gene expression was determined by qPCR. The ability of the synovial cell lines to degrade ECM was assessed by in situ invadosome assays using fluorescent cross-linked gelatine. Confocal microscopy was used to determine the cellular localization of 14-3-3n.

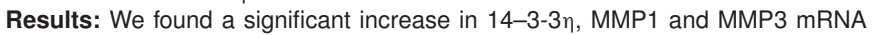
levels in synoviocytes from rheumatoid arthritis patients compared to cells from non-arthritis individuals. A strong correlation between 14-3-3n expression levels and the ability of synoviocyte cell lines to form invadosomes was observed $(r 2=0.8299)$. Knockdown of $14-3-3 \eta$ decreased the ability of arthritic synoviocytes to form invadosomes indicating a role of $14-3-3 \eta$ in extracellular matrix degrading ability. Confocal microscopy revealed that $14-3-3 \eta$ staining was mostly found as small punctated structures in the cytoplasm and at the cell periphery of arthritic synoviocytes where they colocalized with leading edge F-actin and discrete patches of the exocyst component, Exo70.

Conclusions: The finding of the role of $14-3-3 \eta$ in invadosome formation points to a previously unappreciated facet of how 14-3-3n influences joint ECM remodelling and reinforces its role as a marker of RA progression and joint damage. How $14-3-3 \eta$ is involved in the regulation of MMP production/secretion and the possible role it plays in remodelling of actin-rich subcellular structures is the subject of ongoing studies.

Disclosure of Interest: C. Lalanne: None declared, R. Lavoie: None declared, M. Charbonneau: None declared, J. Savill Employee of: Augurex Life Sciences Corp., A. Marotta Employee of: Augurex Life Sciences Corp., C. Dubois: None declared

DOI: 10.1136/annrheumdis-2017-eular.5531

\section{FRI0005 TARGETING CARTILAGE AGING AS OSTEOARTHRITIS THERAPEUTICS BY DRUG REPURPOSING}

B. Carames ${ }^{1}$, U. Nogueira-Recalde ${ }^{1}$, M.I. Loza ${ }^{2}$, F.J. Blanco ${ }^{1}$, E. Dominguez ${ }^{2}$. ${ }^{1}$ Rheumatology Division. Cartilage Biology Group, The Institute of Biomedical Research of A Coruña (INIBIC), A coruña; ${ }^{2}$ Biofarma Research Group, Center for Research in Molecular Medicine and Chronic Diseases (CIMUS), University of Santiago de Compostela, Santiago de Compostela, Spain

Background: Effective treatments for Osteoarthritis $(\mathrm{OA})$ are not available. In aging-related diseases, including $\mathrm{OA}$, failure of cellular homeostasis mechanisms, such as autophagy can cause extracellular matrix destruction and cell death (1). With aging, chondrocyte function is diminished, contributing to a cellular senescence phenotype often observed in OA chondrocytes. In addition, a defect in autophagy is observed in both aging and cartilage degeneration $(2,3)$.

Objectives: The objective of this study was to identify anti-senescence and pro-autophagy molecules by a cell-based high-throughput screening (HTS) in human chondrocytes.

Methods: To induce cellular senescence or reduced autophagy, immortalized human chondrocytes (TC28a2) were seeded (2500 cells/well) in 384 well plates, and treated with IL-6 $(20 \mathrm{ng} / \mathrm{ml})$ for 72 or 18 hours, respectively. Then, chondrocytes were incubated with Prestwick Chemical Library (1120 approved drugs with chemical and pharmacological diversity, as well as bioavailability and safety in humans) at $10 \mu \mathrm{M}$ for 72 hours. To identify anti-senescence hits, nuclei was stained with Hoechst $33342(2,5 \mu \mathrm{g} / \mathrm{ml})$, while $\beta$-galactosidase subcellular structures was stained by using Imagene Green C12FDG substrate ( $30 \mu \mathrm{M})$. To evaluate autophagic flux, a reporter cell line was generated by retrovirus transfection of pBABE-mCherry-EGFP-LC3 plasmid in TC28a2 chondrocytes. Plates were imaged by using Operetta ${ }^{\circledR}$ High Content Screening (HCS) system in non-confocal mode using the 20x WD objective. For each well, 4 fields and 4 planes of bright field, Hoechst and fluorescein channels were obtained. Relative intensity of C12FDG in cytoplasm and number of autophagosomes/autolysosomes per area of cytoplasm were determined to quantitate $\beta$-galactosidase activity and autophagy flux respectively. Compound validation was performed in TC28a2 chondrocytes and in primary human chondrocytes by evaluating cell senescence, autophagy pathway and cell death by apoptosis.

Results: A primary screening was performed to identify anti-senescence compounds by measurement of senescence-associated $\beta$-galactosidase activity. 283 compounds with anti-senescence effects were identified. The anti-senescence hits were analyzed by monitorizing autophagic flux. 29 compounds with both anti-senescence and pro-autophagy effects were selected. Then, one compound was selected for further validation. The compound reduced chondrocyte senescence, increased autophagy $(\mathrm{p}<0.0001)$ and protected against inflammation and cell death by apoptosis in human chondrocytes $(p<0.05)$ in response to IL-6. Interestingly, this protective effect was partially mediated by mTOR inhibition, a proposed mechanism to prevent cartilage aging.

Conclusions: Our screening methodology provides a unique opportunity to repurpose drugs and mechanisms to prevent cartilage aging. Autophagy activation and protection against senescence in chondrocytes may provide benefits for delaying cartilage degeneration.

\section{References:}

[1] Lotz MK and Caramés B. Nat Rev Rheumatol 2011;7:579-587.

[2] Caramés B, et al. Arthritis Rheumatol 2015;67:1568-76.

[3] Caramés B et al. Arthritis Rheum 2010;62:791-801.

Acknowledgements: This study was supported by Instituto de Salud Carlos IIIMinisterio de Economía y Competitividad, Spain-PI14/01324 and Fondo Europeo de Desarrollo Regional (FEDER). 